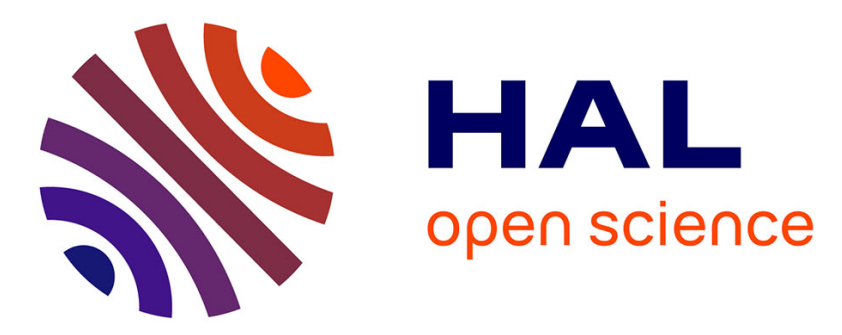

\title{
Nonlinear unitary transformations of space-variant polarized light fields from self-induced geometric-phase optical elements
}

\author{
Nina Kravets, Etienne Brasselet
}

\section{- To cite this version:}

Nina Kravets, Etienne Brasselet. Nonlinear unitary transformations of space-variant polarized light fields from self-induced geometric-phase optical elements. Physical Review A : Atomic, molecular, and optical physics [1990-2015], 2018, 97 (1), pp.13834. 10.1103/PhysRevA.97.013834 . hal-01721480

\author{
HAL Id: hal-01721480 \\ https://hal.science/hal-01721480
}

Submitted on 2 Mar 2018

HAL is a multi-disciplinary open access archive for the deposit and dissemination of scientific research documents, whether they are published or not. The documents may come from teaching and research institutions in France or abroad, or from public or private research centers.
L'archive ouverte pluridisciplinaire HAL, est destinée au dépôt et à la diffusion de documents scientifiques de niveau recherche, publiés ou non, émanant des établissements d'enseignement et de recherche français ou étrangers, des laboratoires publics ou privés. 


\title{
Nonlinear unitary transformations of space-variant polarized light fields from self-induced geometric-phase optical elements
}

\author{
Nina Kravets and Etienne Brasselet* \\ Université Bordeaux, Centre National de la Recherche Scientifique, Laboratoire Ondes et Matière d'Aquitaine, \\ UMR 5798, F-33400 Talence, France
}

(Received 28 October 2017; published 22 January 2018)

\begin{abstract}
We propose to couple the optical orientational nonlinearities of liquid crystals with their ability to self-organize to tailor them to control space-variant-polarized optical fields in a nonlinear manner. Experimental demonstration is made using a liquid crystal light valve that behaves like a light-driven geometric phase optical element. We also unveil two original nonlinear optical processes, namely self-induced separability and nonseparability. These results contribute to the advancement of nonlinear singular optics that is still in its infancy despite 25 years of effort, which may foster the development of nonlinear protocols to manipulate high-dimensional optical information both in the classical and quantum regimes.
\end{abstract}

DOI: 10.1103/PhysRevA.97.013834

\section{INTRODUCTION}

In classical optics, the optical angular momentum of paraxial light fields can be separated into two independent contributions, spin and orbital [1]. The former is associated with the polarization state while the latter refers to the spatial degrees of freedom. The superposition of generalized orthogonal states both for the spin and orbital angular momentum of light leads to a variety of space-variant polarization fields such as cylindrical vector beams [2] or Poincaré beams [3]. Inhomogeneously polarized optical fields have been deployed in many research areas and a nonexhaustive set of examples can be given in focusing [4,5], imaging [6,7], manipulation [8,9], material processing [10,11], nanophotonics [12], laser acceleration of charged particles [13,14], sensing [15,16], and metrology $[17,18]$.

A useful description of such fields in the paraxial limit consists in combining the (spin) Poincaré sphere of polarization [19] with the orbital Poincaré spheres [20], thus leading to a two-dimensional basis constructed from generalized orthogonal states [21-23]. Here we adopt the convention of writing the complex amplitude of a paraxial field propagating along the $z$ axis as being proportional to the propagation factor $\exp \left(-i \omega t \pm i k_{0} z\right)$ with $\omega$ the angular frequency, $t$ the time, and $k_{0}$ the free-space wave vector. In addition, we introduce the spin-orbit optical states $\boldsymbol{\Psi}_{\sigma, \ell}$ defined by the complex vector space-variant polarized light fields

$$
\boldsymbol{\Psi}_{\sigma, \ell}=e^{i \ell \phi} \mathbf{c}_{\sigma} .
$$

In this equation, $\mathbf{c}_{\sigma}=(\mathbf{x}+i \sigma \mathbf{y}) / \sqrt{2}$ with $\sigma= \pm 1$, refers to the circular polarization unit vectors in the Cartesian coordinate system that are associated with $\sigma \hbar$ spin angular momentum per photon [24]. On the other hand, $\exp (i \ell \phi)$ with $\phi=\arctan (y / x)$ and integer $\ell$, refers to a light field associated with $\ell \hbar$ orbital angular momentum per photon [1]. Accordingly, we will consider space-variant polarized light

\footnotetext{
*etienne.brasselet@u-bordeaux.fr
}

fields described by a coherent linear superposition of two states given by Eq. (1), namely,

$$
\boldsymbol{\Psi}\left(\alpha, \beta ; \ell_{+}, \ell_{-}\right)=\alpha \boldsymbol{\Psi}_{+1, \ell_{+}}+\beta \boldsymbol{\Psi}_{-1, \ell_{-}},
$$

where $(\alpha, \beta)$ are complex numbers and $|\alpha|^{2}+|\beta|^{2}=1$. In addition, the present formalism also applies for the arbitrary paraxial radial envelope described by the function $f_{\ell_{+}}(r)=$ $\sum_{p} \alpha_{p} L_{p}^{\left|\ell_{ \pm}\right|}(r)$ where $\alpha_{p}$ are complex coefficients and $L_{p}^{\left|\ell_{ \pm}\right|}$ are the associated Laguerre polynomials.

Many techniques are available to create spatially engineered polarization states. Some rely on simple and efficient schemes, such as those based on interferometry [25], the modal structure associated with fiber optics [26,27], or the use of uniaxial crystals [28]. However, the advent of machining techniques enabling the fabrication of structured optically anisotropic elements has led to novel kinds of optical elements to generate light beams with azimuthally varying linear polarization states from either space-variant true birefringence [29] or spacevariant form birefringence [30]. Spatial light modulators are another kind of device common today having the advantage of remote-controlled operation [31,32]. Optical microcavities have also been designed to support the emission of vector vortex beams [33,34]. In fact, the possibility to generate space-variant polarized beams directly from the source has been investigated in laser physics since the early 1970s by introducing homogeneous anisotropic crystals inside the cavity [35] and nowadays polyvalent laser sources to control spatial features of laser emission have been realized using intracavity inhomogeneous anisotropic optical elements [36].

Remarkably, all the above approaches deal with linear optical processes and, to date, only a few works have explored the use of nonlinear optical processes to generate vector vortex beams. Indeed, it has been shown that a slightly absorbing cubic crystal exhibits self-induced spin-orbit interaction when exposed to an incident linearly polarized continuous-wave Gaussian beam [37]. However, the efficiency of the photoelastic process involved scales quadratically with the incident optical power and is of the order of $10^{-4}$ for $50-\mathrm{W}$ incident power. Also, it has been shown that vector fields can be 
generated by second-harmonic generation from semiconductor nanoantennas by using a focused linearly polarized pulsed Gaussian beam with $\mathrm{mW}$ power level as the incident field [38]. In that case, an efficiency of the order of $10^{-4}$ is obtained, which is a record value for this area of research.

It is worth mentioning other studies dealing with nonlinear optical phenomena involving spin and orbital angular momentum although they do not deal with space-variant polarized fields. Namely, the doubling of the orbital angular momentum per photon by second-harmonic generation in homogeneous nonlinear crystals has been reported $[39,40]$. Second-harmonic generation of orbital angular momentum from a space-variant nonlinear crystal has also been demonstrated [41]. Nonlinear spin-orbit interaction driven by a dc electric field and associated with the electrooptical Pockels effect has been predicted and observed $[42,43]$. However, this effect has a modest $\sim 10 \%$ spin-to-orbital angular momentum conversion efficiency under an applied voltage of the order of $10 \mathrm{kV}$. The case of ponderomotive optical forces is another example where nonlinear generation of orbital angular momentum may occur $[44,45]$. Types of nonlinearities other than those of electronic origin can also be at work. Indeed, the long-range orientational order and high birefringence of liquid crystalline media lead to orientational optical nonlinearities that are several orders of magnitude larger than electronic ones $[46,47]$. In practice, this leads to vivid nonlinear phenomena under continuous-wave radiation and modest power. In the recent years, several nonlinear optics demonstrations involving orbital angular momentum were reported [48-51].

Here we propose to exploit the giant orientational optical nonlinearity of liquid crystals to achieve nonlinear unitary transformations of vector vortex beams using light-driven liquid crystal topological defects. Namely, the idea is to perform optical transformations $\boldsymbol{\Psi}\left(\alpha, \beta ; \ell_{+}, \ell_{-}\right) \rightarrow \boldsymbol{\Psi}\left(\alpha^{\prime}, \beta^{\prime} ; \ell_{+}^{\prime}, \ell_{-}^{\prime}\right)$ with $\left(\alpha, \beta ; \ell_{+}, \ell_{-}\right) \neq\left(\alpha^{\prime}, \beta^{\prime} ; \ell_{+}^{\prime}, \ell_{-}^{\prime}\right)$. By doing so, we extend previous studies on the creation of vector vortex beams from Gaussian beams with uniform linear polarization using linear optics of natural nematic liquid crystal topological defects [52]. Also, this generalizes previous demonstrations of the generation of scalar vortex beams using spin-orbit cross-phase modulation [48] and spin-orbit self-phase modulation [50,51] in nematics. The experimental demonstration is made using a so-called liquid crystal light valve [53] according to an approach detailed in Sec. II, where the characterization and optimization of the nonlinear geometric phase optical element at play is documented. Experimental results are reported and compared to simulations accounting for the nonideal nature of the created nonlinear topological optical element in Sec. III. Then, two specific situations that correspond to nonlinear optical separability and nonseparability processes are discussed in Sec. IV. In particular, we show that the radial degree of freedom cannot be ignored in the reported experiments and its consequences are addressed.

\section{EXPERIMENTAL APPROACH}

\section{A. Principle}

Originally, a q-plate (QP) refers to a type of geometric phase optical element enabling the mappping of the two-dimensional basis associated with the polarization state, $\left(\mathbf{c}_{+}, \mathbf{c}_{-}\right)$, onto a two-dimensional subspace for the orbital angular momentum., $\left(e^{+i \ell \phi}, e^{-i \ell \phi}\right)$ [54]. In practice, a QP is a linear optical element that consists of a space-variant flat retarder satisfying the half-wave plate condition for the used wavelength and ideally characterized by an orientation angle of its in-plane optical axis $\theta=q \phi$, up to an unimportant constant. Neglecting diffraction, it operates the following unitary transformation of an incident circularly polarized plane wave:

$$
\boldsymbol{\Psi}_{\sigma, \ell} \stackrel{\mathrm{QP}}{\longrightarrow} \boldsymbol{\Psi}_{-\sigma, \ell+2 \sigma q} .
$$

Our approach consists in generating a QP in an initially isotropic nonlinear material so that nothing happens at "low" photon flux while a self-induced q-plate (SIQP) is created at "high" photon flux. In the latter case, by combining Eqs. (2) and (3) one gets that a SIQP enables the nonlinear unitary transformation

$$
\boldsymbol{\Psi}\left(\alpha, \beta ; \ell_{+}, \ell_{-}\right) \stackrel{\mathrm{SIQP}}{\longrightarrow} \boldsymbol{\Psi}\left(\beta, \alpha ; \ell_{-}-2 q, \ell_{+}+2 q\right) .
$$

With the aim at realizing the above nonlinear transformation, the present study is based on the possibility to optically induce a spin-dependent optical vortex generator made of a liquid crystal slab associated with perpendicular orientational boundary conditions at the cell walls that define a nematic layer with the typical thickness ranging from 10 to $100 \mu \mathrm{m}$ [48,50,51].

Specifically, it has been shown that the use of liquid crystal light valve [(LCLV), a nematic slab sandwiched between a photoconductive substrate and a standard glass slab, both provided with transparent electrodes] allows almost $100 \%$ efficient self-conversion of an incident circularly polarized Gaussian beam into a scalar optical vortex beam with contracircular polarization state [51]. The underlying phenomenon is the light-driven electrical generation of an umbilical defect with topological charge +1 . Umbilics are electrically induced nonsingular liquid crystal topological structures of charge $m= \pm 1$ appearing in films of nematics having a negative dielectric anisotropy [55]. It has been shown that umbilics behave as a liquid crystal q-plate with $q=m$ [56].

Here we exploit self-induced umbilic q-plates (SIUQPs) created in a liquid crystal light valve by restricting ourselves, without loss of generality, to the case of a "balanced" vectorial optical state characterized by $\alpha=\beta$ and $\ell_{-}=-\ell_{+}=\ell$. This corresponds to linearly polarized vector fields whose electric vector orientation angle has a linear azimuthal dependence, $\psi=\ell \phi$. At the origin, the angle $\psi$ is thus undefined, which defines a vector point singularity ( $V$ point) characterized by a Poincaré-Hopf index [57], here equal to $\ell$, which describes how many turns the electric field vector makes around a full turn nearby the singularity. Adopting the simplified notation $\boldsymbol{\Psi}_{\mathrm{V}}(\ell) \equiv \boldsymbol{\Psi}(1 / \sqrt{2}, 1 / \sqrt{2} ;-\ell, \ell)$, the SIUQP thus ideally operates the following transformation only if the incident photon flux is high enough:

$$
\boldsymbol{\Psi}_{\mathrm{V}}(\ell) \stackrel{\substack{\text { SIUQP } \\ \text { ideal }}}{\longrightarrow} \Psi_{\mathrm{V}}(2-\ell) .
$$

\section{B. Experimental setup}

We use the setup shown in Fig. 1. The key element is the LCLV that consists of a $L=34-\mu$ m-thick layer of the nematic 


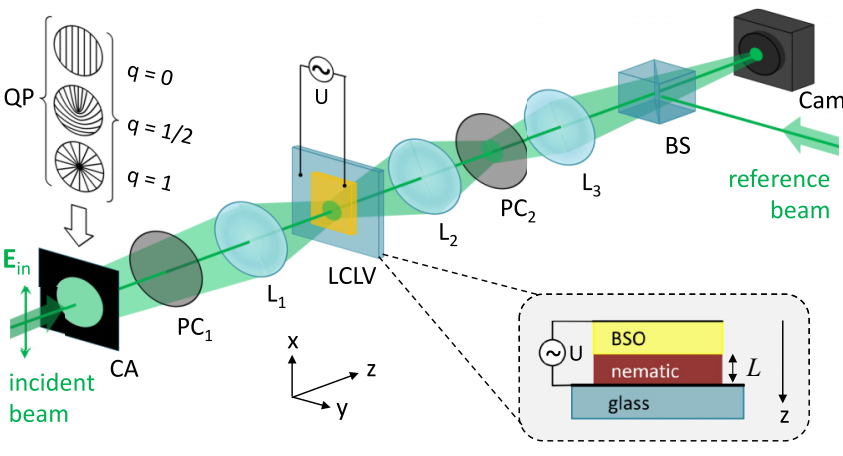

FIG. 1. Experimental setup. $\mathbf{E}_{\text {in }}$ : linearly polarized incident Gaussian beam; CA clear circular aperture with diameter $3 \mathrm{~mm} ; \mathrm{PC}_{i}$ : polarization controllers; $L_{1}$ : lens with focal length $f_{1}=100 \mathrm{~mm}$; LCLV: liquid crystal light valve whose enlargement depicts its structure (see text for details); $U$ : applied voltage on the LCLV. $L_{2}$ and $L_{3}$ : lenses with focal length $f_{2}=f_{3}=75 \mathrm{~mm}$; BS: beamsplitter; Cam: camera.

liquid crystal mixture MLC6608 (Merck) characterized by a negative relative dielectric permittivity $\epsilon_{a} \simeq-4.2$ at $1 \mathrm{kHz}$ frequency and room temperature. This last is sandwiched between a 1-mm-thick slab of bismuth silicon oxide (BSO) photoconductive crystal and a 1-mm-thick glass slab which are both provided with transparent electrodes. The inset of Fig. 1 depicts the location of the electrodes on which is applied an ac electric field at $2-\mathrm{kHz}$ frequency. The inner walls of the cell are treated with cetyl-trimethyl-ammonium-bromide surfactant that provides uniform molecular orientation of the liquid crystal along the $z$ axis at rest.

We prepare a set of five incident states $\boldsymbol{\Psi}_{\mathrm{V}}(\ell)$ with $-2 \leqslant$ $\ell \leqslant 2$ by using solid-state QPs made of nanostructured glass slabs with $q=(0,1 / 2,1)$ illuminated by a Gaussian laser beam linearly polarized along the $x$ beam at wavelength $\lambda=532 \mathrm{~nm}$. Indeed, noting that $\mathbf{x}=\left(\mathbf{c}_{+}+\mathbf{c}_{-}\right) / \sqrt{2}$, Eq. (3) gives

$$
\mathbf{x} \stackrel{\mathrm{QP}}{\longrightarrow} \boldsymbol{\Psi}_{\mathrm{V}}(2 q) .
$$

In our case, this gives an access to $\ell=(0,1,2)$. The negative values of $\ell$ are then obtained without need of additional $\mathrm{q}$ plates by inserting a half-wave plate (HWP, which is ensured by the polarization controller $P C_{1}$ ) after the QP. This ensures the transformation $\boldsymbol{\Psi}_{\mathrm{V}}(\ell) \stackrel{\text { HWP }}{\longrightarrow} \boldsymbol{\Psi}_{\mathrm{V}}(-\ell)$. In practice, a 3-mmdiameter clear circular aperture (CA) is placed in the plane of the QP, where the incident Gaussian beam waist diameter is $\simeq 4 \mathrm{~mm}$. The last plane is reimaged onto the nematic layer with a magnification factor of 0.2 using the lens $L_{1}$. Polarimetric analysis of the output light field is performed by reimaging the LCLV using the lens $L_{2}$ placed in a $2 f-2 f$ configuration with respect to the polarization controller $P C_{2}$. The field is eventually reimaged on a camera (Cam) with a magnification factor of 3.25 using the lens $L_{3}$. The spatial features of the phase are also assessed by interfering the output field with a reference Gaussian beam owing to the beamsplitter BS, which is removed during the polarization study.

The experimental protocol consists in applying a fixed voltage $U \simeq 0.9 U_{\text {th }}$, where $U_{\text {th }} \simeq 7 \mathrm{~V}_{\text {rms }}$ refers to the threshold value below which the nematic layer remains unperturbed in

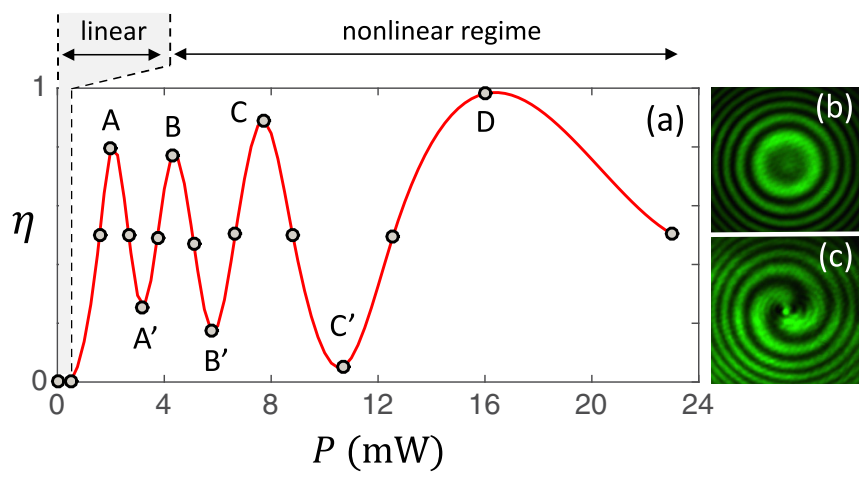

FIG. 2. (a) Power dependence of the parameter $\eta$ that characterizes the self-induced generated scalar vortex field for an incident circularly polarized unstructured light. Markers: measurements for incident power giving extremal values of $\eta$ as well as $\eta \simeq 0.5$. Solid line: spline fit of the experimental data. Interference pattern between the output field and a reference Gaussian beam in the linear and nonlinear regimes in panels (b) and (c), respectively. The second of these refers to the fourth maximum of $\eta(P)$, which corresponds to the realization of a SIUQP. Labels A to D refer to local maxima occurring at $P=2,4.3,7.7$, and $16 \mathrm{~mW}$, see Fig. 3 while labels $\mathrm{A}^{\prime}$ to $\mathrm{C}^{\prime}$ refer to local minima occurring at $P=3.2,5.8$, and $10.7 \mathrm{~mW}$.

the absence of light, and illuminating the LCLV according to Fig. 1 with total optical power $P$ impinging on the sample. At first, we check the ability of the LCLV to act as a SIUQP in the case of a circularly polarized incident unstructured field onto the sample. This is done by setting $q=0$ and $P C_{1}$ as a quarter-wave plate oriented at $\pm 45^{\circ}$ from the $x$ axis. The efficiency of the process is evaluated by measuring the fraction of the total output power carried by the contra-circularly polarized component, $\eta$, using $P C_{2}$ either as right-handed or left-handed circular polarizer. Its dependence as a function of the incident power is shown in Fig. 2(a).

At low power $(P \lesssim 0.5 \mathrm{~mW})$ the output field is not affected by the LCLV that behaves as a linear, isotropic, and homogeneous optical medium. This is illustrated in Fig. 2(b) showing the usual concentric fringes pattern in the presence of a coaxial Gaussian reference beam. Above a threshold power, the liquid crystal reorients and forms an umbilical defect. In return, a scalar optical vortex beam with topological charge \pm 2 is generated. This corresponds to the nonlinear regime characterized by an oscillation of $\eta(P)$. The fact that near-unit value can be reached [see label D, Fig. 2(a)] proves that a SIUQP can be readily obtained. Accordingly, the output field is fully transformed into a scalar vortex beam, as shown in Fig. 2(c) where the two-arm spiral pattern is the indication of the topological charge 2 .

\section{Self-induced umbilic q-plate characteristics}

Basically, there is a two-fold distinction between a SIUQP and an ideal QP. First, the birefringent phase retardation $\Delta$ is no longer uniform in the $(x, y)$ plane of the sample. An indirect signature of it can be grasped from Fig. 2(a). In contrast to a QP exhibiting oscillation of $\eta$ between 0 and 1 as the uniform retardance increases [58], here the overall monotonic increase of the liquid crystal orientation with power leads to extremal 
(a) XCPOL $\leftrightarrow$ information on $\Delta(r)$

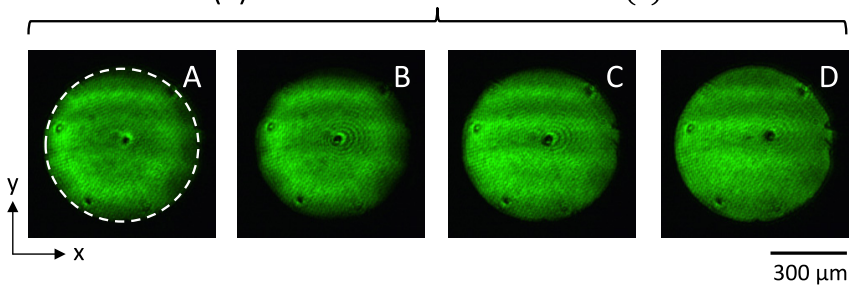

(b) XLPOL $\leftrightarrow$ information on $\psi(r)$

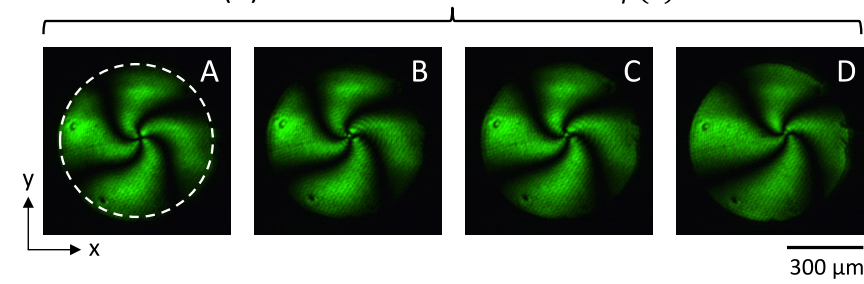

FIG. 3. (a) Images of the LCLV observed between crossed circular polarizers at incident optical power values that correspond to local maxima for the scalar optical vortex generation as reported in Fig. 2(a), see labels A to D. (b) Same as in (a) using crossed linear polarizers. The dashed white circles refer to the geometric image of CA.

values for $\eta$ that are neither 0 nor 1 . This is explained by the fact that the optically localized umbilical defect structure is unavoidably accompanied by a radially varying retardance. Namely, $\Delta=0$ at the defect core and $\Delta \rightarrow 0$ outside the illumination area since $U<U_{\mathrm{th}}$. Therefore, neither the condition $\Delta=2 n \pi$ [i.e., $\eta=0$ ] nor $\Delta=(2 n+1) \pi$ [i.e., $\eta=1$ ] $(n>0$ integer) can be satisfied uniformly. The present case is thus the optical analog of the electrically driven localized umbilic QP studied in a previous work [59]. Still, as already noticed in the previous subsection, $\eta \simeq 1$ can be reached at larger power [see label D, Fig. 2(a)].

In practice, direct information on the retardance can also be obtained by imaging the LCLV placed between crossed circular polarizers (XCPOL) using the excitation light only, see Fig. 3(a) where each panel refers to a local maximum of $\eta$ as indicated by the labels A to D. Indeed, within the paraxial approximation and neglecting diffraction effects as well as nonadiabatic propagation inside the structure, XCPOL images are associated with intensity profiles $I_{\text {input }}(x, y) \sin ^{2}[\Delta(x, y) / 2]$ with $I_{\text {input }}(x, y)$ the intensity profile at the input facet of the sample [60]. Therefore, the black dots in Fig. 3(a) refer to the umbilic core with zero retardance. On the other hand, the circular contour sharpening and the improved uniformity of the intensity distribution as the power increases from the case A to D indicate the establishment of almost uniform light-driven retardance inside the geometric image of the input circular aperture.

The second characteristic distinguishing a SIUQP from an ideal QP relates to the spatial distribution of the optical axis orientation. Indeed, $\theta$ is no longer solely dependent on the azimuthal angle $\phi$ as is the case for a QP. This is illustrated in Fig. 3(b) where crossed linear polarizers (XLPOL) images of the LCLV are shown. The four dark brushes are the signature of an umbilic with unit strength [61], however, the observed axisymmetric swirl around the defect core indicates a radial contribution to the space-variant optical axis $\theta=\phi+\varphi(r)$.
The function $\varphi(r)$ characterizes the swirl of the light-driven umbilic that has been identified to result from the elastic anisotropy of the nematic liquid crystal [62]. Interestingly we notice that the swirl tends to decrease as the power increases, as one can see from the evolution from the case A to D in Fig. 3(b).

From the above analysis of the spatial features of both the retardance and optical axis orientation of the SIUQP, we fix the power to $P=16 \mathrm{~mW}$ when working in the nonlinear regime. This choice suggests a simple, yet relevant, model to describe the SIUQP. Namely, we assume the birefringent phase retardance to be uniform and equal to an odd value of $\pi$ uniform inside the geometric image of the input circular aperture. Since $P=16 \mathrm{~mW}$ corresponds to the fourth maximum of $\eta(P)$, we set $\Delta=7 \pi$. Also, we neglect the observed off-axis defect core, see Fig. 3, that results from the competition between various distinct contributions to the total torque density exerted on the liquid crystal [51]. Eventually, the function $\theta(r, \phi)$ is experimentally assessed by Stokes polarimetry as detailed below.

When the incident light is circularly polarized, the orientation of the major axis of the total output field polarization ellipse $\psi$ is oriented at $\pm 45^{\circ}$ from the effective optical axis orientation. We thus have $\psi=\theta \pm \pi / 4$, the \pm sign being dictated by the incident polarization handedness and the value of the retardance [63]. The experimental map $\theta_{\exp }$ is thus retrieved by exploiting the standard polarimetry relationship $\psi=(1 / 2) \arctan \left[\left(I_{45^{\circ}}-I_{135^{\circ}}\right) /\left(I_{0^{\circ}}-I_{90^{\circ}}\right)\right]$ [64], where $I_{\alpha}$ refers to the intensity profile obtained by placing a polarizer at an angle $\alpha$ from the $x$ axis at the output of the film, see Fig. 4(a). The result is shown in Fig. 4(b) where the presence of noise outside the geometric image of the input circular aperture (dashed circle) is due to the absence of signal in that region. The $\theta_{\exp }$ map confirms the topological charge +1 of the SIUQP and its swirled character. Moreover, this allows extraction of the radial dependence of the swirl according to the relationship $\varphi_{\exp }=\theta_{\exp }-\phi$. This is done by defining a circular region of interest of radius $R$ around the defect core taken as the coordinate origin, see the solid white circle in Fig. 4(b). A series of measurements for a representative sample of 180 equally spaced values of $\phi$ is shown in Fig. 4(c), where we discarded the noisy data close to the origin, typically for $r<R / 10$. Their mean value (thick cyan solid line) is then adjusted by an exponentially decaying function (red solid line)

$$
\varphi(r)=\varphi_{0}+\varphi_{1}\left(1-e^{-r / r_{\mathrm{c}}}\right)
$$

with $\varphi_{0}=-0.805, \varphi_{1}=1.849$, and $r_{\mathrm{c}}=0.385 R$, which gives a satisfying agreement. We note that the obtained value for the characteristic swirl length, $r_{\mathrm{c}} \simeq 80 \mu \mathrm{m}$, is of the order of the cell thickness as expected from the nonlocal character of a liquid crystal film.

In summary, the SIUQP obtained in our experiments can be quantitatively described by $\Delta=7 \pi$ and $\theta(r, \phi)=\phi+\varphi(r)$. This model will be used in the next section to simulate our experimental attempts to realize the nonlinear transformations described by Eq. (5).

\section{NONLINEAR TRANSFORMATIONS OF $\Psi_{\mathrm{V}}$}

Following the approach presented in Sec. II B we prepare a set of incident optical states $\boldsymbol{\Psi}_{\mathrm{V}}(\ell)$ with $-2 \leqslant \ell \leqslant+2$ that 

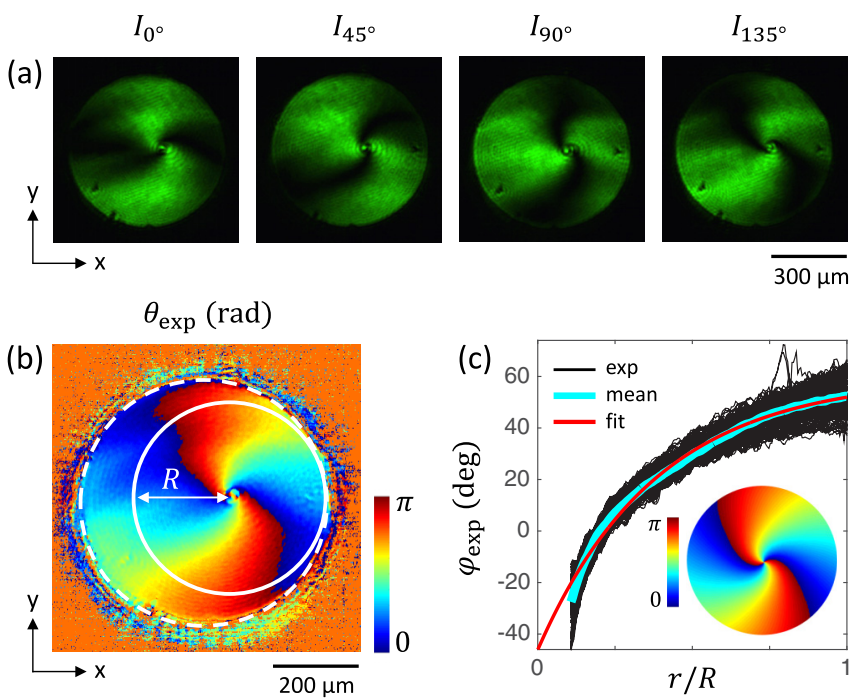

FIG. 4. (a) Images of the LCLV illuminated by a incident circularly polarized light, adjusting the polarization controller $P C_{2}$ as a polarizer oriented at an angle $\alpha=0^{\circ}, 45^{\circ}, 90^{\circ}$, and $135^{\circ}$ from the $x$ axis. (b) Experimentally reconstructed map $\theta_{\exp }$ of the effective optical axis orientation of the SIUQP. Dashed white circle: contour of the geometric image of the circular aperture CA. Solid white circle: region of interest used to evaluate experimentally the swirl function $\varphi_{\exp }(r)$. (c) Determination of the swirl function $\varphi_{\exp }(r)$ according to the protocol described in the text. The inset displays the modeled map $\theta(r, \phi)$ of the optical axis orientation of the SIUQP over the region of interest.

are individually sent onto the LCLV. The experimental maps $\psi_{\exp }$ of the output electric field vector orientation in the region of interest are collected in Fig. 5(a) for the linear regime. The comparison to the expected maps associated with $\boldsymbol{\Psi}_{\mathrm{V}}(\ell)$ is shown in Fig. 5(b), which agree well with the data. Still one must also check the degree of linear polarization (DOLP),

(a) Output $\psi_{\text {exp }}$ maps : linear regime

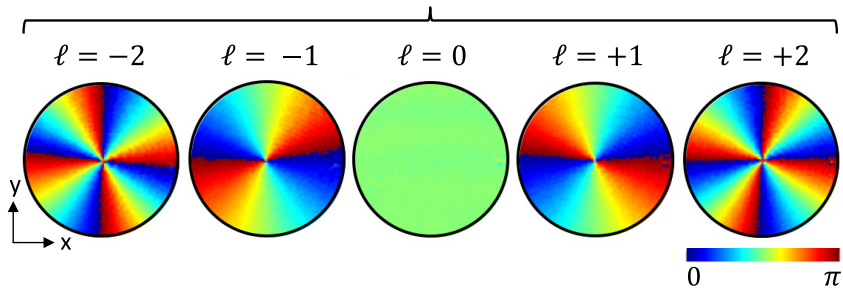

(b) Simulations associated with $\boldsymbol{\Psi}_{\mathrm{V}}(\ell)$

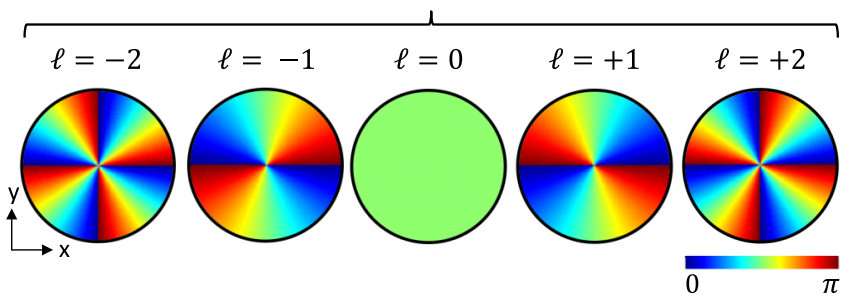

FIG. 5. (a) Experimental output maps $\psi_{\exp }$ of the electric field vector orientation in the linear regime for input optical states $\boldsymbol{\Psi}_{\mathrm{V}}(\ell)$ with $-2 \leqslant \ell \leqslant+2$. (b) Simulated maps associated with $\boldsymbol{\Psi}_{\mathrm{V}}(\ell)$.
TABLE I. Average value of the degree of linear polarization (DOLP) in the linear and nonlinear regimes.

\begin{tabular}{lccccc}
\hline \hline$\ell$ & -2 & -1 & 0 & +1 & +2 \\
\hline DOLP (linear regime) & 0.92 & 0.93 & 0.99 & 0.93 & 0.91 \\
DOLP (nonlinear regime) & 0.88 & 0.92 & 0.93 & 0.91 & 0.89 \\
\hline \hline
\end{tabular}

which equals 1 for any state $\boldsymbol{\Psi}_{\mathrm{V}}(\ell)$. The evaluation is made from the relationship DOLP $=\left(s_{1}^{2}+s_{2}^{2}\right)^{1 / 2}$ where $s_{1}$ and $s_{2}$ are the first and second reduced Stokes parameters that can be evaluated from the previously defined intensity profiles $I_{\alpha}$. The results are summarized in Table I and complete the linear regime analysis.

In the nonlinear regime, however, the measurements do not correspond to the output state $\boldsymbol{\Psi}_{\mathrm{V}}(2-\ell)$ given by Eq. (5), as one can see by comparing Fig. 6(a) to Fig. 6(b). Indeed, twisted maps are observed in all the cases except for $\ell=+2$, though the latter remains associated with radial nonuniformity. Such a behavior is explained by the swirled nature of the SIUQP that imparts an additional geometric phase contribution to the output field. Noticeably, this happens in a differential manner for the two orthogonal components $\boldsymbol{\Psi}_{+,-\ell}$ and $\boldsymbol{\Psi}_{-,+\ell}$ of the input state. This can be derived by Jones calculus accounting

(a) Output $\psi_{\text {exp }}$ maps : nonlinear regime

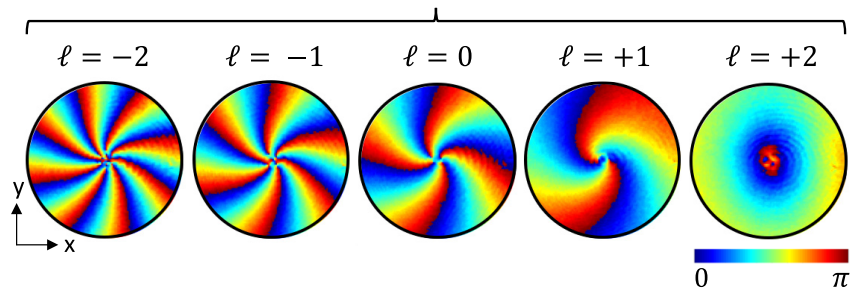

(b) Simulations associated with $\Psi_{\mathrm{V}}(2-\ell)$

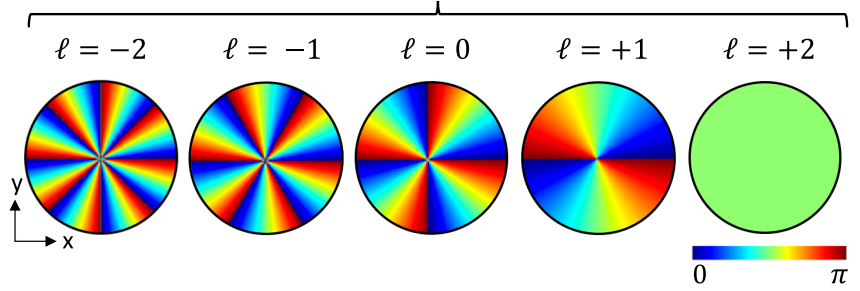

(c) Simulations associated with $\widetilde{\boldsymbol{\Psi}}_{\mathrm{V}}(2-\ell)$

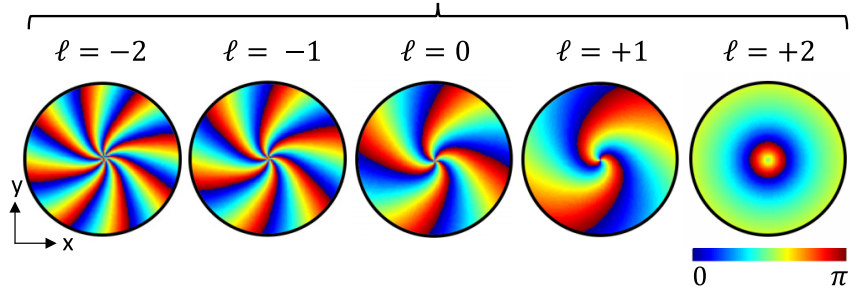

FIG. 6. (a) Experimental output maps $\psi_{\exp }$ of the electric field vector orientation in the nonlinear regime for input optical states $\boldsymbol{\Psi}_{\mathrm{V}}(\ell)$ with $-2 \leqslant \ell \leqslant+2$. (b) Simulated maps associated with $\boldsymbol{\Psi}_{\mathrm{V}}(2-\ell)$. (c) Simulated maps associated with the hybrid states $\widetilde{\boldsymbol{\Psi}}_{\mathrm{V}}(2-\ell)$, see text for details. 
for the above-retained description of the SIUQP, which allows us to identify that a hybrid vectorial state has been generated. Namely, one gets

$$
\boldsymbol{\Psi}_{\mathrm{V}}(\ell) \stackrel{\substack{\text { SIUQP } \\ \text { real }}}{\longrightarrow} \widetilde{\Psi}_{\mathrm{V}}(2-\ell),
$$

where

$$
\widetilde{\boldsymbol{\Psi}}_{\mathrm{V}}(2-\ell)=\frac{1}{\sqrt{2}}\left[e^{-2 i \varphi(r)} \boldsymbol{\Psi}_{+, \ell-2}+e^{2 i \varphi(r)} \boldsymbol{\Psi}_{+, 2-\ell}\right]
$$

The evaluation of the $\psi$ maps for the latter hybrid state is shown in Fig. 6(c), which gives a good agreement when compared to the observations in Fig. 6(a).

Note that any state $\boldsymbol{\Psi}_{\mathrm{V}}(\ell)$ with $\ell \neq 0$ has the property of being nonseparable: neither the spin nor the orbital degree of freedom can be factorized. As such, any attempt to measure one of the two degrees of freedom is perturbing the other. This finds many classical optics applications as well as being a source of mutual inspiration with its quantum analog (quantum entanglement) see, for instance, a recent discussion in Ref. [65]. In the present case, the nonseparable state is perturbed by the involvement of the radial degree of freedom, which has, nevertheless, an interesting application potential, as reported in Ref. [66] in a linear optics experiment using a static solid-state vortex lens. In the next section we address the issue of nonlinear (non)separability, which is an original feature of our experiments.

\section{NONLINEAR (NON)SEPARABILITY}

Since $\boldsymbol{\Psi}_{\mathrm{V}}(\ell)$ with $\ell \neq 0$ is a nonseparable state for the spin and orbital degrees of freedom while $\boldsymbol{\Psi}_{\mathrm{V}}(0)$ is separable, we notice two particular cases of interest. Indeed, the SIUQP performs a nonlinear optical nonseparability operation when $\ell=0$ while the separable analog occurs when $\ell=+2$. Such a nonlinear "mixing" or "demixing" of the spin and orbital degree of freedom can be directly assessed by observing the interference patterns resulting from the coherent superposition between each of the circularly polarized components of the output field and a copolarized coaxial reference Gaussian beam. The results are summarized in Fig. 7.

In the linear regime, the incident states are unperturbed as demonstrated in the previous section, hence one recovers circular fringes for $\ell=0$ and a two-arm spiral with opposite handedness for $\ell=+2$. On the other hand, when the nonlinearity is at play, the nonlinear regimes evidence the generation of two orthogonal orbital states with charge $-\sigma_{\text {out }}(2-\ell)$ where $\sigma_{\text {out }}= \pm 1$ refers to the helicity components of the output field. (a) Linear regime

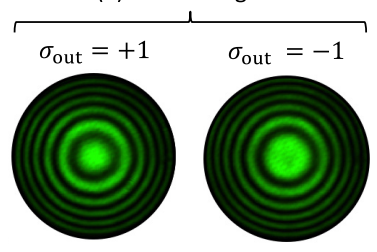

(c) Linear regime
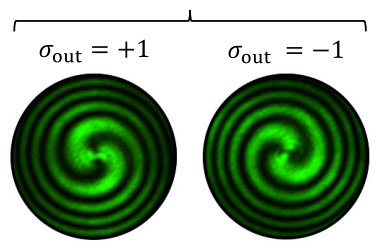

(b) Nonlinear regime

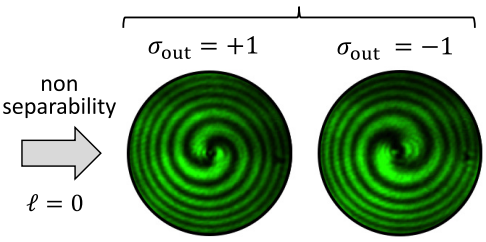

FIG. 7. $(\mathrm{a}, \mathrm{b})$ Nonlinear optical nonseparability when $\ell=0$. Interference pattern between the $\mathbf{c}_{\sigma_{\text {out }}}$-polarized components of the output field with a copolarized reference Gaussian beam in the (a) linear and (b) nonlinear regimes. (c,d) Same as in $(\mathrm{a}, \mathrm{b})$ but for nonlinear optical separability when $\ell=+2$.

\section{CONCLUSION AND OUTLOOK}

While the use of geometric-phase optical elements to manipulate the orbital angular momentum degree of freedom has become common, there are only rare demonstrations of nonlinear attempts to control it. Here we propose to use liquid crystals, which are prime choice materials in the development of linear optical elements to process the orbital angular momentum of freedom of light, to realize fully efficient nonlinear unitary transformations of superpositions of generalized orthogonal states involving both the spin and orbital degrees of freedom. The demonstration is made by exploiting two key properties of liquid crystals: their giant optical orientational nonlinearities and their ability to self-organize into topological defects. In addition, we note that although the demonstration is made at a single wavelength, the generalization to other wavelengths is straightforward since the liquid crystal light valve retardance can be tuned with the incident optical power. By proposing an efficient route to transform vector vortex beams via a nonlinear interaction between light and matter, these results enrich the nonlinear singular optics toolbox. We anticipate the elaboration of nonlinear protocols to manipulate high-dimensional spinorbit optical states, and hence agile manipulation of the optical information.

\section{ACKNOWLEDGMENT}

This study has been carried out with financial support from the French National Research Agency (Project No. ANR-15CE30-0018).
[1] L. Allen, M. W. Beijersbergen, R. J. C. Spreeuw, and J. P. Woerdman, Orbital angular momentum of light and the transformation of Laguerre-Gaussian laser modes, Phys. Rev. A 45, 8185 (1992).
[2] Q.Zhan, Cylindrical vector beams: From mathematical concepts to applications, Adv. Opt. Photon. 1, 1 (2009).

[3] A. M. Beckley, T. G. Brown, and M. A. Alonso, Full Poincaré beams, Opt. Express 18, 10777 (2010). 
[4] R. Dorn, S. Quabis, and G. Leuchs, Sharper Focus for a Radially Polarized Light Beam, Phys. Rev. Lett. 91, 233901 (2003).

[5] Y. Kozawa and S. Sato, Sharper focal spot formed by higher-order radially polarized laser beams, J. Opt. Soc. Am. A 24, 1793 (2007).

[6] K. Moh, X.-C. Yuan, J. Bu, S. Zhu, and B. Z. Gao, Radial polarization induced surface plasmon virtual probe for twophoton fluorescence microscopy, Opt. Lett. 34, 971 (2009).

[7] G. Bautista, M. J. Huttunen, J. Makitalo, J. M. Kontio, J. Simonen, and M. Kauranen, Second-harmonic generation imaging of metal nano-objects with cylindrical vector beams, Nano Lett. 12, 3207 (2012).

[8] Q. Zhan, Trapping metallic Rayleigh particles with radial polarization, Opt. Express 12, 3377 (2004).

[9] Y. Kozawa and S. Sato, Optical trapping of micrometer-sized dielectric particles by cylindrical vector beams, Opt. Express 18, 10828 (2010).

[10] M. Meier, V. Romano, and T. Feurer, Material processing with pulsed radially and azimuthally polarized laser radiation, Appl. Phys. A 86, 329 (2007).

[11] J. Hamazaki, R. Morita, K. Chujo, Y. Kobayashi, S. Tanda, and T. Omatsu, Optical-vortex laser ablation, Opt. Express 18, 2144 (2010).

[12] M. Neugebauer, T. Bauer, P. Banzer, and G. Leuchs, Polarization tailored light driven directional optical nanobeacon, Nano Lett. 14, 2546 (2014).

[13] W. Kimura, G. Kim, R. Romea, L. Steinhauer, I. Pogorelsky, K. Kusche, R. Fernow, X. Wang, and Y. Liu, Laser Acceleration of Relativistic Electrons Using the Inverse Cherenkov Effect, Phys. Rev. Lett. 74, 546 (1995).

[14] Y. I. Salamin, Z. Harman, and C. H. Keitel, Direct High-Power Laser Acceleration of Ions for Medical Applications, Phys. Rev. Lett. 100, 155004 (2008).

[15] R. Wang, C. Zhang, Y. Yang, S. Zhu, and X.-C. Yuan, Focused cylindrical vector beam assisted microscopic pSPR biosensor with an ultra wide dynamic range, Opt. Lett. 37, 2091 (2012).

[16] S. Berg-Johansen, F. Töppel, B. Stiller, P. Banzer, M. Ornigotti, E. Giacobino, G. Leuchs, A. Aiello, and C. Marquardt, Classically entangled optical beams for high-speed kinematic sensing, Optica 2, 864 (2015).

[17] F. K. Fatemi, Cylindrical vector beams for rapid polarizationdependent measurements in atomic systems, Opt. Express 19, 25143 (2011).

[18] F. Töppel, A. Aiello, C. Marquardt, E. Giacobino, and G. Leuchs, Classical entanglement in polarization metrology, New J. Phys. 16, 073019 (2014).

[19] H. Poincaré, Théorie Mathématique de la Lumière, Vol. 2, (Gauthiers-Villars, Paris, 1892).

[20] M. J. Padgett and J. Courtial, Poincaré-sphere equivalent for light beams containing orbital angular momentum, Opt. Lett. 24, 430 (1999).

[21] G. Milione, H. I. Sztul, D. A. Nolan, and R. R. Alfano, HigherOrder Poincaré Sphere, Stokes Parameters, and the Angular Momentum of Light, Phys. Rev. Lett. 107, 053601 (2011).

[22] A. Holleczek, A. Aiello, C. Gabriel, C. Marquardt, and G. Leuchs, Classical and quantum properties of cylindrically polarized states of light, Opt. Express 19, 9714 (2011).

[23] X. Yi, Y. Liu, X. Ling, X. Zhou, Y. Ke, H. Luo, S. Wen, and D. Fan, Hybrid-order Poincaré sphere, Phys. Rev. A 91, 023801 (2015).
[24] R. A. Beth, Mechanical detection and measurement of the angular momentum of light, Phys. Rev. 50, 115 (1936).

[25] S. C. Tidwell, D. H. Ford, and W. D. Kimura, Generating radially polarized beams interferometrically, Appl. Opt. 29, 2234 (1990).

[26] T. Grosjean, D. Courjon, and M. Spajer, An all-fiber device for generating radially and other polarized light beams, Opt. Commun. 203, 1 (2002).

[27] G. Volpe and D. Petrov, Generation of cylindrical vector beams with few-mode fibers excited by Laguerre-Gaussian beams, Opt. Commun. 237, 89 (2004).

[28] T. A. Fadeyeva, V. G. Shvedov, Y. V. Izdebskaya, A. V. Volyar, E. Brasselet, D. N. Neshev, A. S. Desyatnikov, W. Krolikowski, and Y.S. Kivshar, Spatially engineered polarization states and optical vortices in uniaxial crystals, Opt. Express 18, 10848 (2010).

[29] M. Stalder and M. Schadt, Linearly polarized light with axial symmetry generated by liquid-crystal polarization converters, Opt. Lett. 21, 1948 (1996).

[30] Z. Bomzon, V. Kleiner, and E. Hasman, Formation of radially and azimuthally polarized light using space-variant subwavelength metal stripe gratings, Appl. Phys. Lett. 79, 1587 (2001).

[31] M. A. Neil, F. Massoumian, R. Juškaitis, and T. Wilson, Method for the generation of arbitrary complex vector wave fronts, Opt. Lett. 27, 1929 (2002).

[32] C. Maurer, A. Jesacher, S. Fürhapter, S. Bernet, and M. RitschMarte, Tailoring of arbitrary optical vector beams, New J. Phys. 9, 78 (2007).

[33] X. Cai, J. Wang, M. J. Strain, B. Johnson-Morris, J. Zhu, M. Sorel, J. L. OBrien, M. G. Thompson, and S. Yu, Integrated compact optical vortex beam emitters, Science 338, 363 (2012).

[34] S. A. Schulz, T. Machula, E. Karimi, and R. W. Boyd, Integrated multi vector vortex beam generator, Opt. Express 21, 16130 (2013).

[35] D. Pohl, Operation of a ruby laser in the purely transverse electric mode $\mathrm{TE}_{01}$, Appl. Phys. Lett. 20, 266 (1972).

[36] D. Naidoo, F. S. Roux, A. Dudley, I. Litvin, B. Piccirillo, L. Marrucci, and A. Forbes, Controlled generation of higher-order Poincaré sphere beams from a laser, Nat. Photon. 10, 327 (2016).

[37] S. Mosca, B. Canuel, E. Karimi, B. Piccirillo, L. Marrucci, R. De Rosa, E. Genin, L. Milano, and E. Santamato, Photon selfinduced spin-to-orbital conversion in a terbium-gallium-garnet crystal at high laser power, Phys. Rev. A 82, 043806 (2010).

[38] R. Camacho-Morales, M. Rahmani, S. Kruk, L. Wang, L. Xu, D. A. Smirnova, A. S. Solntsev, A. Miroshnichenko, H. H. Tan, F. Karouta, S. Naureen, K. Vora, L. Carletti, C. de Angelis, C. Jagadish, Y. S. Kivshar, and D. N. Neshev, Nonlinear generation of vector beams from AlGaAs nanoantennas, Nano Lett. 16, 7191 (2016).

[39] I. Basistiy, V. Y. Bazhenov, M. Soskin, and M. V. Vasnetsov, Optics of light beams with screw dislocations, Opt. Commun. 103, 422 (1993).

[40] K. Dholakia, N. Simpson, M. Padgett, and L. Allen, Secondharmonic generation and the orbital angular momentum of light, Phys. Rev. A 54, R3742 (1996).

[41] D. Wei, Y. Zhu, W. Zhong, G. Cui, H. Wang, Y. He, Y. Zhang, Y. $\mathrm{Lu}$, and M. Xiao, Directly generating orbital angular momentum in second-harmonic waves with a spirally poled nonlinear photonic crystal, Appl. Phys. Lett. 110, 261104 (2017).

[42] L. Chen and W. She, Electro-optically forbidden or enhanced spin-to-orbital angular momentum conversion in a focused light beam, Opt. Lett. 33, 696 (2008). 
[43] I. Skab, Y. Vasylkiv, I. Smaga, and R. Vlokh, Spin-to-orbital momentum conversion via electro-optic Pockels effect in crystals, Phys. Rev. A 84, 043815 (2011).

[44] M. Beresna, P. G. Kazansky, Y. Svirko, M. Barkauskas, and R. Danielius, High average power second harmonic generation in air, Appl. Phys. Lett. 95, 121502 (2009).

[45] D. Gordon, B. Hafizi, and A. Ting, Nonlinear conversion of photon spin to photon orbital angular momentum, Opt. Lett. 34, 3280 (2009).

[46] N. Tabiryan, A. Sukhov, and B. Y. Zel'Dovich, Orientational optical nonlinearity of liquid crystals, Mol. Cryst. Liq. Cryst. 136, 1 (1986).

[47] I. C. Khoo, Nonlinear optics of liquid crystalline materials, Phys. Rep. 471, 221 (2009).

[48] E. Brasselet, Spin-orbit optical cross-phase-modulation, Phys. Rev. A 82, 063836 (2010).

[49] F. Lenzini, S. Residori, F. Arecchi, and U. Bortolozzo, Optical vortex interaction and generation via nonlinear wave mixing, Phys. Rev. A 84, 061801 (2011).

[50] M. E. Ketara and E. Brasselet, Self-induced nonlinear spin-orbit interaction of light in liquid crystals, Opt. Lett. 37, 602 (2012).

[51] R. Barboza, U. Bortolozzo, G. Assanto, E. Vidal-Henriquez, M. G. Clerc, and S. Residori, Vortex Induction Via Anisotropy Stabilized Light-Matter Interaction, Phys. Rev. Lett. 109, 143901 (2012).

[52] C. Loussert, U. Delabre, and E. Brasselet, Manipulating the Orbital Angular Momentum of Light at the Micron Scale with Nematic Disclinations in a Liquid Crystal Film, Phys. Rev. Lett. 111, 037802 (2013).

[53] P. Aubourg, J. P. Huignard, M. Hareng, and R. A. Mullen, Liquid crystal light valve using bulk monocrystalline $\mathrm{Bi}_{12} \mathrm{SiO}_{20}$ as the photoconductive material, Appl. Opt. 21, 3706 (1982).

[54] L. Marrucci, C. Manzo, and D. Paparo, Optical Spin-to-Orbital Angular Momentum Conversion in Inhomogeneous Anisotropic Media, Phys. Rev. Lett. 96, 163905 (2006).
[55] A. Rapini, Umbilics: Static properties and shear-induced displacements, J. Phys. 34, 629 (1973).

[56] E. Brasselet and C. Loussert, Electrically controlled topological defects in liquid crystals as tunable spin-orbit encoders for photons, Opt. Lett. 36, 719 (2011).

[57] I. Freund, Polarization singularity indices in Gaussian laser beams, Opt. Commun. 201, 251 (2002).

[58] B. Piccirillo, V. DAmbrosio, S. Slussarenko, L. Marrucci, and E. Santamato, Photon spin-to-orbital angular momentum conversion via an electrically tunable q-plate, Appl. Phys. Lett. 97, 241104 (2010).

[59] C. Loussert, K. Kushnir, and E. Brasselet, Q-plates micro-arrays for parallel processing of the photon orbital angular momentum, Appl. Phys. Lett. 105, 121108 (2014).

[60] E. Brasselet, Tunable Optical Vortex Arrays from a Single Nematic Topological Defect, Phys. Rev. Lett. 108, 087801 (2012).

[61] A. Rapini, L. Léger, and A. Martinet, Umbilics: Static and dynamic properties, J. Phys. Colloques 36, 189 (1975).

[62] R. Barboza, U. Bortolozzo, M. G. Clerc, S. Residori, and E. Vidal-Henriquez, Light-matter interaction induces a single positive vortex with swirling arms, Philos. Trans. R. Soc. London A 372, 20140019 (2014).

[63] Note that the measurement requires $\Delta \neq n \pi, n$ integer, which implies detuning the incident power from its optimal value $P=$ $16 \mathrm{~mW}$ for that measurement.

[64] M. Born and E. Wolf, Principles of Optics (Pergamon, London, 2005).

[65] S. M. H. Rafsanjani, M. Mirhosseini, O. S. Magaña-Loaiza, and R. W. Boyd, State transfer based on classical nonseparability, Phys. Rev. A 92, 023827 (2015).

[66] A. M. Tam et al., Bifocal Optical-Vortex Lens with Sorting of the Generated Nonseparable Spin-Orbital Angular-Momentum States, Phys. Rev. Appl. 7, 034010 (2017). 